\title{
Die Historische Karte der Schweiz von Kümmerly \& Frey 1971/72
}

\author{
Georges Grosjean
}

Im Jahre 1970 faßte der Verlag Kümmerly \& Frey den Entschluß, die in ihrer Bearbeitung über ein halbes Jahrhundert alte und vergriffene Schulwandkarte der Schweiz von Prof. Dr. Wilhelm Oechsli und A. Baldamus durch eine neue Karte zu ersetzen. Dabei kam der Gedanke auf, dieselbe Karte in reduziertem Maßstab von 1:500 000 auch als Handkarte herauszubringen, in Ansehen, daß zurzeit weder für die Schule noch für die Wissenschaft eine im Handel erhältliche historische Karte der alten Eidgenossenschaft vorliegt. Die ausgezeichnete Karte zur territorialen Entwicklung der Schweizerischen Eidgenossenschaft von Adolf Gasser und Ernst Keller vom Jahre 1932, seinerzeit erschienen als Beilage zu Heft 4 der «Schweizer Kriegsgeschichte», herausgegeben im Auftrage des Chefs des Generalstabs, und selbständig mit ausführlichem Kommentar, ist nur noch in Bibliotheken vorhanden und recht selten geworden. Im Historischen Atlas der Schweiz von Hektor Ammann und Karl Schib (1951/58) sind nur Gesamtkarten der Schweiz in kleineren Maßstäben von Adolf Gasser und nach ganz uneinheitlichen Gesichtspunkten bearbeitete historische Karten der Kantone enthalten, die zusammen kein Bild der Gesamteidgenossenschaft ergeben. Ebenso wurden für den Atlas der Schweiz nur kleinmaßstabige historische Kärtchen verschiedener Zustände der alten Eidgenossenschaft entworfen.

Für den Bearbeiter der neuen Karte stellten sich somit einige interessante Probleme. Zuerst mußte über die Zielsetzung Klarheit gewonnen werden. Es sollte eine Karte geschaffen werden, die im selben Generalisierungsgrad als Wandkarte nicht überladen ist und anderseits als Handkarte genügend hergibt, um für Schule und Wissenschaft ein wertvolles Hilfsmittel zu sein. Der Benützerkreis für die Handkarte mußte anderseits aus Kostengründen möglichst groß konzipiert werden. Vom Verlag aus bestand der Wunsch, möglichst rasch wieder zu einer historischen Wandkarte der Schweiz zu kommen. Es konnte daher zur Bearbeitung nicht unbegrenzt Zeit für neue wissenschaftliche Grundlagenforschung und die Organisation eines großen «teamwork» von Regionalhistorikern vorgesehen werden. Die bestehenden Karten konnten nicht übernommen und revidiert werden. Die Karte von Oechsli unterscheidet in ihren Farben nur die Kategorien nicht einmal die Territorien der einzelnen Orte der alten Eidgenossenschaft und ist dazu rein statisch, indem sie den Zustand gegen Ende des 18. Jahrhunderts gibt. Eine solche grob verallgemeinernde Darstellung hätte den Anforderungen an eine heutige Wandkarte knapp, denjenigen an eine Handkarte gar nicht entsprochen. Die Karte von Gasser und Keller (1932) dagegen wäre als Wandkarte viel zu feingliedrig, und außerdem sind ihre Farben ausschließlich nach Phasen des Zuwachses verwendet, also dynamisch, so daß die territoriale Gliederung der alten Eidgenossenschaft, die vor allem für die Schule und die unteren Stufen der Wissenschaft im Vordergrund stehen muß, nicht zum Ausdruck kommt. Die Karte, an sich hervorragend, ist für einen bestimmten Zweck konzipiert und richtet sich an einen recht anspruchsvollen und eher kleinen Benützerkreis.

Es ergaben sich daraus für den Bearbeiter folgende Entschlüsse, die von der Verlagsleitung gebilligt wurden:

- Die Karte ist von Grund auf neu zu konzipieren. Zur Bearbeitung muß vorwiegend auf die bestehende Literatur und die bestehenden Karten abgestellt werden.

- Die Karte soll sich an einen Benützerkreis richten, der von der höhern Mittelschule über den interessierten Nichthistoriker bis zum allgemeinen Gebrauch der Wissenschaft reicht unter Ausschluß einer eigentlichen Popularisierung einerseits, wie auch spezieller Bedürfnisse der Fachwissenschaft anderseits.

- Die Karte soll sowohl statische (Zustand) wie auch dynamische Aspekte (Wachstum) berücksichtigen, da sie grundsätzlich allein, nicht in einer Serie anderer Zustandskarten benützt wird (Wandkarte). Aus Gründen der Übersichtlichkeit und Verständlichkeit soll aber der statische Aspekt (Zustand der alten Eidgenossenschaft gegen Ende des 18. Jahrhunderts) optisch dominieren.

- Die Wandkarteninhalte sollen als kräftige, gut trennende Farbflächen und wenige große Signaturen das Kartenbild auf Distanz beherrschen und einfach erscheinen lassen. Bei der Nahbetrachtung als Handkarte oder auch als Wandkarte soll 
ein angemessener Detailreichtum die Karte wertvoll machen. Insbesondere sollen die für die Lokalgeschichte interessanten internen Einteilungen der Orte und Zugewandten, Landvogteien, Gerichtsbezirke, Kastlaneien, Ämter und ihre Verwaltungszentren, ferner Schlösser, Ruinen, Klöster, Schlachtfelder, Bäder, Landsgemeindeplätze und andere historische Stätten in genügender Zahl in Erscheinung treten.

- Die Zahl der Druckfarben wurde in Abschätzung der Erfordernisse der Darstellung und des angestrebten niederen Preises der Karte auf fünf festgelegt: Schwarz, Grau, Rot, Gelb, Blau.

Die nun vorliegende fertige Karte dürfte zeigen, daß die Ziele erreicht worden sind. Ende 1971 konnte die Wandkarte 1: 200000 ausgeliefert werden. Im Frühjahr 1972 wurde die Handkarte 1:500 000 gedruckt und kann hier den Abonnenten der Geographica Helvetica überreicht werden. Im Handel wird die Karte mit einem ausführlichen Kommentarheft in deutscher und französischer Sprache abgegeben. Es ist ein farbenfrohes, harmonisches Kartenbild entstanden. Die klare Unterordnung der Handkarteninhalte unter die großen Flächen und Signaturen der Wandkarte dürfte geglückt sein. Die Territorien der einzelnen Orte sind durch die unterschiedlichen Farben mit einem Blick erfaßbar. Es ist geradezu grotesk, daß diese so naheliegende Darstellungsart hier in einer Karte der alten Eidgenossenschaft vorher noch nie angewendet wurde. Alle Gesamtkarten der alten Eidgenossenschaft mit Ausnahme derjenigen von Gasser-Keller (1932) konnten sich bis in die Gegenwart vom Darstellungsschema Oechslis nicht lösen und verwenden ein und dieselbe Farbe für alle dreizehn Orte, höchstens daß die Länderorte mit einem intensiveren Raster belegt sind. In unserer Karte lassen die Rasterabstufungen der einzelnen Farben vier große Phasen des Wachstums erfassen, allerdings weniger für die Gesamtheit der Eidgenossenschaft als für den einzelnen Ort. So springt z. B. sofort in die Augen, daß Zürich um 1415 erst etwa die Hälfte seines Territoriums beisammen hat, während Bern sein deutsches Land bis auf wenige Ergänzungen abgerundet hat. Dagegen verzeichnet Zürich in der Phase 1416

PS: Als Beilage zu Heft 3/1972 überreicht der Verlag Kümmerly \& Frey AG Bern den Lesern der Geographica Helvetica ein Exemplar "Historische Karte der Schweiz» in der Ausführung als Handkarte. Für dieses wertvolle Geschenk sei ihm der verbindlichste Dank ausgesprochen. Die Redaktion bis 1515 einen großen Gebietszuwachs, während Bern in seiner Ausdehnung fast stagniert - und dies im dynamischen Zeitalter des Burgunderkrieges!

Demgegenüber läßt die Karte wieder erkennen, daß nach 1516 Bern und Freiburg in der Westschweiz erst ihre größte Expansionsphase durchmachten, während die übrige Eidgenossenschaft saturiert ist. Kartographie zwingt immer zu eindeutigen Aussagen. Darin kann für historische Karten einige Gefahr liegen, indem manches klar erscheint, das in Wirklichkeit nichts weniger als klar ist oder war. So können die Flächenfarben der Orte eine gewisse einheitliche Gebietshoheit vortäuschen, die eher moderner Staatskonzeption entspricht. In Wirklichkeit waren die Befugnisse der Städte oder regierenden Länder in den einzelnen Teilen ihres Staatswesens sehr verschiedenartig. Man unterscheidet: Landeshoheit (wozu u. a. das Militäraufgebot gehörte), hohes Gericht, niederes Gericht, Herrschaftsrechte (Twing und Bann) und kirchliche Befugnisse. Nicht immer waren alle Kompetenzen in einer Hand vereinigt. Die wichtigsten Fälle territorialer Überschneidungen sind in der Karte durch Streifenkolorit angedeutet, um etwa dem Lehrer Gelegenheit zu geben, diese Verhältnisse an typischen Beispielen zu illustrieren: so die abwechselnde hohe Gerichtsherrschaft Berns und der Bischöfe von Basel auf dem Tessenberg, das hohe Gericht Berns im solothurnischen Bucheggberg, das hohe Gericht Zürichs im Kelleramt, dessen niedere Gerichte und Herrschaftsrechte bei der Stadt Bremgarten lagen, die Herrschaftsrechte und niederen Gerichte Zürichs in einzelnen Herrschaften des Thurgaus.

Die Bearbeitung wurde viel zeitraubender als angenommen, indem die Ungleichheit der kartographischen Vorlagen erst in den Einzelheiten zum Vorschein kam. Was bedeutet "Erwerbung» eines Gebietes durch einen eidgenössischen Ort? Ist es Erwerbung der Landeshoheit mit der hohen Gerichtsbarkeit? In den meisten Fällen wurde dieses Kriterium angenommen und versucht, eine gewisse Einheitlichkeit herzustellen. Es gibt aber Fälle, wo die Erwerbung der Grundherrschaft mit Twing und Bann, niederem Gericht, Bodenzins- und Vogteieinkünften oder bei Klöstern gar noch reichen Zehnteinkünften für den Stadtstaat unvergleichlich interessanter war als das hohe Gericht, das im Jahr höchstens einige Fälle abzuurteilen gab. Einige kantonale historische Karten nehmen daher den Erwerb der Grundherrschafts- oder Vogteirechte als Zeitpunkt der Zugehörigkeit zu einem Kanton an. Das gibt bisweilen große Unterschiede in der Jahreszahl der Erwerbung auf verschiedenen kursierenden Karten. Da und dort haben wir in unserer Karte auf das Problem hingewiesen, indem in Streifenkolorit in zwei Rasterabstufungen die zeitliche Staffelung der Erwerbung angedeutet wurde. So 
etwa die luzernischen Herrschaften Büron und Wikon, über welche Luzern die hohen Rechte 1407 mit der Grafschaft Willisau erwarb, aber in deren effektiven Besitz die Stadt erst 1455 bzw. 1476 durch Erwerb der Grundherrschaftsrechte aus der Hand ausländischer Adelsgeschlechter gelangte. Ähnlich wurde in Graubünden angedeutet, daß der größte Teil des Zehngerichtebundes und das Unterengadin zwar 1497/99 mit den drei Bünden den Anschluß an die Eidgenossenschaft vollzogen, daß aber Österreich in diesen Gebieten noch bedeutende Rechte besaß, die erst im 17. Jahrhundert nach den Bündner Wirren abgelöst wurden.

Auf diese Weise deutet die Karte mindestens an, in welcher Richtung die historische Wirklichkeit zu suchen ist. Es galt hier, zwischen der verwirrenden Vielfalt der tatsächlichen Verhältnisse und dem Bedürfnis nach klarer und einfacher Darstellung den optimalen Standort $\mathrm{zu}$ finden. Daß dieser Standort näher bei der Generalisierung liegt, ist durch den ursprünglich primären Verwendungszweck als Wandkarte bedingt.

Adresse des Verfassers:

Prof. Dr. Georges Grosjean

Geogr. Institut der Universität, Abt. für angewandte Geographie

Effingerstraße 4, 3000 Bern 